\title{
The Buffering Effect of Supervisor Support on the Relationship between Perceived Stress and Turnover Intention among Female Nurses in Saudi Arabia
}

\author{
Dr. Sitah Alshutwi, PhD, RN.
}

Associate Dean, Clinical Affairs

Assistant Professor; Nursing Management; King Saud bin Abdul Aziz University for Health Sciences (KSAU-HS)

Ministry of National Guard Health Affairs, Central Region; KSAU-HS

*Corresponding author: Dr. Sitah Alshutwi; shutwis@ksau-hs.edu.sa

Received 03 May 2021;

Accepted 26 May 2021;

Published 04 June 2021

\begin{abstract}
Background: The shortage of registered nurses is a worldwide concern; with many countries struggling to maintain adequate numbers of nurses. Many factors that contribute to nursing shortage have been studied, but the influence of work-family conflict has received little attention. Workfamily conflict has been linked to a number of work-related consequences, including turnover intention among employees. The aim of this study was to evaluate the influence of supervisory support behaviours on the relationship among work-family conflict, Stress, and turnover intention. Sample: 113 nurses. Result: A significant positive correlation was found between work-family conflict and turnover intention. A negative correlation was found between supervisory support behaviours and turnover intention. Both work-family conflict and stress were associated with turnover intention; however, these associations were buffered (weaken), when nurses perceived higher supervisory support behaviours. Conclusion Supervisory support behaviours could be the tool to help married, female nurses play their professional role without compromising their family responsibilities. Nurses' turnover is a complex issue that may require multiple prevention strategies; however, enhancing family supportive supervisor behaviors could be a key resource for maintaining a positive workplace environment and reducing turnover intention.
\end{abstract}

Keywords: Turnover, Stress, Nurses

\section{Introduction}

Many countries around the world are struggling to maintain adequate number of nurses (WHO, 2010). Inadequate nursing staffing could compromise the quality of patient care (Moore, 2014). Among many factors that contribute to RN turnover, the influence of work-family conflict (WFC) has gained little attention (Leineweber, et al., 2014). In Saudi Arabia, the turnover rate among Saudi nurses reached $50 \%$ of the total employed nurses (Abu Zinadah, 2010). Work-family conflict was found to be a reason that influence Saudi nurses to leave their workplace (Alonazi, \& Omar, 2013).

Not only WFC was found to be significantly association with increased turnover intention among employees (Amstad, Meier, Fasel, Elfering, Semmer, 2011),WFC has been linked to a number of negative consequences, including lower job satisfaction and organizational commitment (Hoobler \& Wilson, 2010), sleep insufficiency, insomnia symptoms (Crain, et al., 2014), obesity (Grzywacz, 2000), cardiovascular diseases (Shockley \& Allen, 2013), sleep insufficiency (Berkman, Buxton, Ertel, \& Okechukwu, 2010), and high cholesterol (Greenhaus et al., 2006). Many behavioral, psychological, and career scholars have studied the role of supervisor support on reducing the consequences of WFC (Kossek et al., 2011; Shockley \&Allen, 2013). Family Supportive Supervisor Behaviors (FSSB) has been defined as a set of behaviors exhibited by supervisors that show support of employees' family roles. FSSB has been found to be a promising approach contributing to the reduction of turnover intention with employees' who experience WFC (Kalidass \& Bahron, 2015; Nohe $\&$ Sonntag, 2014). However, limited studies were conducted in the nursing profession. Further, it was found that $75 \%$ the samples used in work-family studies are exclusively US workers (Eby, Casper, Lockwood, Bordeaux, Brinley, 2005). No studies were found that included a sample from Saudi Arabia. Therefore, the purpose of this study was to evaluate the influence of FSSB on the relationship among WFC, Stress, and turnover intention within the culture context of Saudi.

\section{Background}

Managing work and family demands is a challenge for many employees including nurses (Kelly et al., 2014). Researchers have 
moved beyond examining the causes of WFC to study the effects of WFC (Almeida, et al., 2016). Stress has been presented as a reaction of exposure to higher WFC. Individuals who were more reactive were found to be more subject to negative sequences than individuals who were less reactive (Piazza, Charle, Sliwinski, Mogel, Almeida, 2013; Mroczek et al., 2015). Therefore, Almeida, et al, (2016) recommended measuring the level of stress when studying WFC. Employees who experienced higher WFC, also reported higher level of stress (Almeida, et al., 2016; Li, Shaffer, \& Bagger, 2015). Stress, in turn, has been linked to longitudinal negative consequences such as depression, anxiety (Charles et al., 2013), chronic diseases (Piazza, Charles, Sliwinski, Mogle, \& Almeida, 2013), and even mortality (Mroczek et al., 2015).

Although stress has been presented as a reaction or consequence to WFC, there is a potential of opposite temporal sequences of the direction of the relationship between WFC and stress. Due to the use of cross-sectional designs by many researchers, insight into the direction of this relationship could not be provided. Many authors argue that experiencing higher WFC could increase the level of stress (Buxton, Klein, Whinnery, Williams, \& McDade, 2013; Kreiner, 2006), other argue that being stressed could increase the level of WFC (Li, Shaffer, \& Bagger, 2015).

Employees may try to reduce the conflict or stress by quitting their job (Nohe, \& Sonntag, 2014). In an effort to efficiently manage WFC and reduce employee turnover, many behavioral, psychological, and career scholars have focused on the role of supervisors (Kossek et al., 2011; Shockley \&Allen, 2013). A growing body of researchers have documented the benefits of FSSB on employee health (Hammer, Kossek, Anger, Bodner, \& Zimmerman, 2011).

FSSB facilitate an employee's desire to effectively balance and manage work and family responsibilities. Supervisor behaviors include engaging in emotional support, instrumental support, rolemodeling behaviors, and exploring creative work-family management practices (Hammer, Kossek, Yragui, Bodner, \& Hanson, 2009). This set of supervisor behaviors that were supportive of employees' family roles was identified as a promising approach to reduce employees' turnover intention (TI) (Kalidass \& Bahron, 2015; Nohe \&Sonntag, 2014).

Few studies have examined the buffering effect of FSSB on turnover intention. Nohe and Sonntag (2014), in a study of 665 employees concluded that FSSB buffered the relationship between WFC and turnover intention. This finding suggests that FSSB need further examination as a potential effective strategy to reduce turnover intention for those who are experience high WFC. According to social support theories and the buffering hypothesis (House, 1981), individuals who perceive high levels of support are more able to manage stressful situation such as WFC and reduce its negative consequences such as turnover intention (Cohen, Gottlieb, \& Underwood, 2000).

Despite the importance of work-family issues and the influence of FSSB, limited studies were conducted among nurses and limited studies examined the influence of FSSB on the relationships among WFC, stress and turnover intention. Further, it was found that $75 \%$ of work-family studies that have been conducted used samples of only US workers (Eby, Casper, Lockwood, Bordeaux, Brinley, 2005). No published studies were found that included a sample from Saudi Arabia. Therefore, this study was conducted in one of the major hospitals in Riyadh city, the capital of Saudi Arabia.

\section{Culture Context of Saudi Arabia}

Women in Saudi Arabia, as many women in the Gulf regions countries, have high levels of education and many work opportunities; yet they are expected, according to cultural norms, to perform traditional domestic responsibilities, bear children and look after their family needs (Bahudhailah, 2015; Shallal, 2011). According to customs and mores in the Saudi society, marriage and motherhood are highly valued (Mobaraki \& Söderfeldt, 2010), and women are not expected to prioritize work over family and children. For working women, these expectations can create conflict between work and family demands (Sha'aban, 1996).

Unlike men in Western countries who currently are expected to perform a larger domestic role (Parker \&Wang, 2013; Coyle, Van Leer, Schroeder, \& Fulcher, 2015), most men in Gulf regions, especially in Saudi Arabia, do not participate in domestic responsibilities. Due to their culture, they heavily relay on their wives who are expected to takes care of almost all domestic house work and responsibilities regardless whether they are working outside the home or not (Shallal, 2011; Alyaemni, et al., 2013). For many married women, this situation is a serious burden, it is hard to meet the needs of both work and family roles without facing a conflict and experiencing stress (Shallal, 2011).

Many married women in the Gulf region countries, especially in Saudi Arabia, were found to be significantly dissatisfied with their employment (Shallal, 2011). The difficulty of balancing family and work responsibilities was a serious factor that influenced women's participation in the general workforce (Aryee et al., 1999). There is a common understanding that work could diminish the quality of family life (Nazzah, 2004), therefore, it is not surprising to find that the rates of single women were higher than married women in general workforce in Arab countries including Saudi Arabia (Fargues, 2005).

According to the Saudi society, emphasized by both norms and cultural traditions, while a married woman is required to fulfill her family obligations at home, the man's responsibility is to financially support the wife and the family (Metcalfe, 2008). Traditionally and culturally, Men have been the family providers and with the current oil revenues in the area, the need for women to work after marriage is minimized as the family can depend on husband income alone (Shallal, 2011). However, Saudi women like many women around the world, choose to work not only to be financially independent but also to find a sense of purpose and fulfillment (Alyaemni, et al., 2013; Lal, 2012).

With the defined roles of married women and men in the Saudi society, married working women are more vulnerable to experience WFC and stress (Al-Tarrah, 2010; Krouse, and Afifi, 2007; Vanagas et al, 2004). Since the priority for women is home, husband and children, many may choose to quit their jobs to stay homes and fulfill their traditional domestic role (Alyaemni, et al., 2013). Leaving their job could be an easier option for women after marriage, especially when they start experiencing increased WFC. Women in Saudi Arabia would chose not to work if their work would compromise their obligations and responsibilities toward their families (Elamin, \& Omair. 2010).

Although there is an increasing recognition of the role of culture in WFC, the majority of research on WFC has been conducted in Western countries (Aryee, Luk, Leung, \& Lo, 1999; Poelmans, 2005). Little is known about the influence of culture in Gulf region countries, especially Saudi Arabia, on working women. Married women in Saudi Arabia, are seeking a workplace where they can perform their professional duties without compromising their obligatory family responsibilities (Alyaemni, et al., 2013). From this cultural perspective of women placing a strong value on family, FSSB could be expected to influence the relationships 
between WFC and turnover intention especially among married working women (Weisgram et al., 2011).

\section{Purpose}

The purpose of this study was to assess the moderating effect of FSSB on the relationships among WFC, Stress, and turnover intention in Saudi Arabian female registered nurses. In this study, it is proposed that FSSB would weaken the relationship between WFC and turnover intention especially for nurses who have a higher level of stress. It is also proposed that this interaction is determined by marital status; since married nurses may feel more obligated to their family and spouses. Therefore, the main hypothesis for this study was that FSSB moderate (buffer) the relationship between WFC and turnover intention, such the relationship is stronger for married nurses with high (vs. low) stress level.

\section{Method}

A cross-sectional correlational study design was used for this study. Pen and paper self-report questionnaires was used to gather the data from female registered nurses providing direct health care. After optiaint the IRB approval (SP16/013) from King Abdullah International Medical Research Center (KAIMRC), a total of 200 surveys were distributed to Saudi nurses working in one of the largest hospital in the capital city of Saudi Arabia, and 113 were returned for a response rate of $56 \%$. The average age of the nurses was 28 years old (SD 3.8), $47 \%$ of the total sample was married. The majority of the nurses in this sample had a Bachelor Degree in nursing (96\%); and $61 \%$ were working for 12 hours a day, four days a week.

\section{Instruments}

For this study, four instruments with a total of 37 items were used along with some demographic question (age, marital status, years of employment, number of children, age of youngest child, working hours, experience as RN, education, and units). The four measurement scales include:

The perceived stress scale (PSS-10). (Cohen, Kamarck \& Mermelstein, 1983) was used to measure nurses' stress. It is a 10item inventory using a 5-point Likert Scale, ranging from 0 (Never) to 4 (Very Often). A total score of perceived stress (0-40) was calculated for each nurse and higher scores indicate higher levels of perceived stress (Cuneo et al., 2011). The internal consistency estimate for the scale in this study was 0.72 .

The turnover intention scale (TIS) developed by Cammann, Fichman, Jenkins, \& Klesh (1979) was used to measure nurses' intention to leave. The TIS is a 3-item scale with response options ranging from (1) "Extremely Disagree" to (5) "Extremely Agree Rationale. The internal consistencies for the TIS scale in this study was 0.65 .

The work-family conflict scale (Netemeyer, Boles, \& McMurrian, 1996) measured the nurses' level of WFC. Ten items are rated on a scale from 1 (Strongly Disagree) to 5 (Strongly Agree). Higher scores indicate higher levels of conflict. The WFC scale had an internal consistency was 0.88 in this study sample.

The Family supportive supervisor behavior (FSSB) developed by Hammer et al. (2009) was used to measure nurses' perception of supervisors' behavior. It is a 14 items scale and included four dimensions: emotional support (4 items), role modeling behaviors (3 items), instrumental support ( 3 items), and creative work-family management (4 items). The internal consistencies for the scale in this study sample was 0.97 .

\section{Analyses}

Basic descriptive and bivariate correlations were calculated for initial data description. An independent-samples t-test was conducted to compare the level of turnover intention among married and unmarried nurses. The main analysis employed hierarchical multiple regression with turnover intention as the dependent variable and WFC, Stress, and FSSB as independent variables in order to examine the influence of FSSB on the relationships among WFC, stress, and turnover intention. Since it was proposed that the moderation effect of FSSB would be different based on the marital status of nurses, Data were stratified by marital status: married and unmarried.

In order to reduce multicollinearity, the variables were mean-centered before computing the interaction terms (Aiken \& West, 1991). In the first step, turnover intention was entered as the dependent variable, and predictors WFC, FSSB and Stress were entered as independent variables. In Step 2 of the regression analysis, the three two-way interactions (WFC*Stress; WFC*FSSB; Stress*FSSB) were entered. In the last step, the threeway interaction was entered (WFC*Stress*FSSB) into the equation. To confirm the moderation hypotheses, the coefficient for the interaction variables needed to be significant and in the direction predicted.

\section{Results}

More than half of the nurses (68\%) reported having high level of WFC, and $44 \%$ reported having high level of stress. Fifty percent of the total sample reported intentions to leave employment and $34.5 \%$ perceived that they received low support from their direct supervisors. Correlations between study variables are presented in Table 1. There was a significant difference in the level of turnover intention for married $(\mathrm{M}=3.24, \mathrm{SD}=1.04)$ and unmarried nurses $(\mathrm{M}=2.8, \mathrm{SD}=1.07)$, [t (109) $=2.0, \mathrm{p}=0.047]$; suggesting that married Saudi nurses had higher levels of turnover intention than unmarried.

Table 1: Mean scores, standard deviations, and correlations between study variables. $(n=113)$

\begin{tabular}{|l|l|l|l|l|l|l|}
\hline & Mean & SD & TI & WFC & Stress & FSS \\
\hline TI & 3.02 & 1.0 & 1 & & & \\
\hline WFC & 3.42 & .81 & $.44^{* * *}$ & 1 & & \\
\hline Stress & 2.97 & .48 & $.37^{* *}$ & $.475^{* *}$ & 1 & \\
\hline FSS & 3.36 & .97 & $-.53^{* *}$ & $-.31^{* *}$ & $-.32^{* *}$ & 1 \\
\hline
\end{tabular}

**. Correlation is significant at the 0.01 level (2-tailed).

For this study, a hierarchal regression analysis was performed using married nurses $(\mathrm{n}=51)$ with turnover intention as the dependent variable to examine the interaction between FSSB, WFC and stress. In the first step, the three main variables were entered and explained $28.9 \%$ of the variance in turnover intention. In the second step, the bivariate interaction variables were added explaining an additional $7.7 \%$ of the variance in the turnover intention. In the third step, the three-way interaction was added and significantly explained an additional $7 \%$ of the variance in the turnover intention for a total of $43.5 \%$ of the variance of turnover intention explained (Table 2). 
Table 2: Hierarchal regression results for married nurses. $(\mathbf{n}=\mathbf{5 1})$

\begin{tabular}{|l|l|l|l|}
\hline & Step 1 & Step 2 & Step 3 \\
\hline & B & B & $\boldsymbol{\beta}$ \\
\hline Main effect & & & \\
WFC & .174 & -1.24 & $-3.56^{* *}$ \\
Stress & .147 & -.809 & $-2.54^{* *}$ \\
FSS & $-.381^{* *}$ & $-.534^{* *}$ & $-.500^{* *}$ \\
\hline 2-Way interaction terms & & & \\
WFC*Stress & & 1.99 & $5.28^{* *}$ \\
WFC*FSS & & $.296^{*}$ & $.425^{* *}$ \\
Stress*FSS & & -.066 & .279 \\
\hline 3-Way interaction term & & & $-.543^{*}$ \\
WFC*Stress*FSS & & & \\
\hline$\Delta \mathrm{R}^{2}$ & $.289 * *$ & .07 & $.070^{*}$ \\
\hline
\end{tabular}

$F(7,44)=4.843 p<.0005 R^{2}=.435$

* Beta is significant at the 0.05 level

**Beta is significant at the 0.01 level

For unmarried nurses $(\mathrm{n}=58)$, another hierarchal regression analysis was performed with turnover intention as the dependent variable to examine the interaction between FSSB, WFC and stress. In the first step, the main effect explained $45 \%$ of the variance in turnover intention. In the second step, the bivariate interaction explained an additional $9.6 \%$ of the variance in turnover intention. In the third step, the three-way interaction was entered and explained an additional three percent of the variance in turnover intention for a total of $59 \%$ of the variance in the turnover intention explained (Table 3).

Table 3: Hierarchal regression results for unmarried nurses. $(\mathbf{n}=58)$

\begin{tabular}{|l|l|l|l|}
\hline & Step 1 & Step2 & Step 3 \\
\hline & $\mathrm{B}$ & $\mathrm{B}$ & $\beta$ \\
\hline Main effect & & & \\
WFC & $.333^{* *}$ & -.368 & .056 \\
Stress & .040 & -.483 & -.145 \\
FSS & $-.475^{* *}$ & $-.587 * *$ & $-.664 * *$ \\
\hline
\end{tabular}

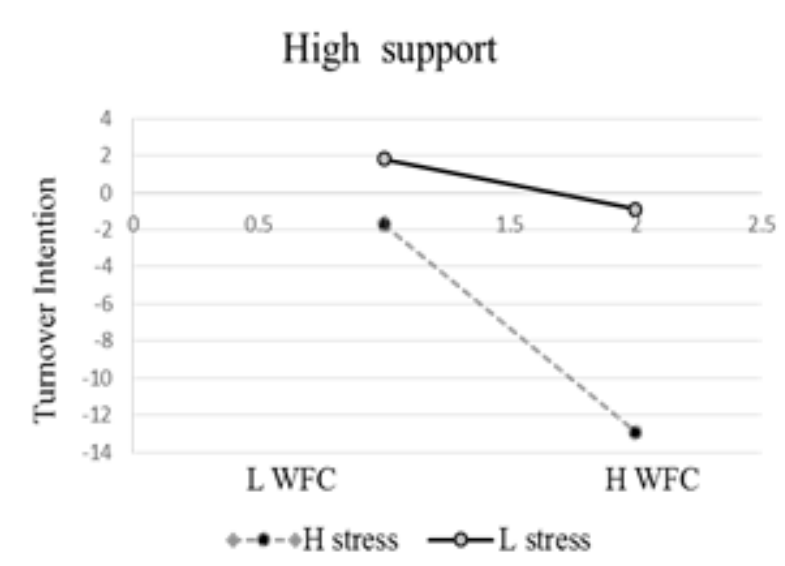

\begin{tabular}{|l|l|l|l|}
\hline 2-Way interaction terms & & & \\
WFC*Stress & & .970 & $.467 * *$ \\
WFC*FSS & & $.372 * *$ & $.325^{* *}$ \\
Stress*FSS & & -.219 & -.192 \\
\hline 3-Way interaction term & & & \\
WFC*Stress*FSS & & & $.281 *$ \\
\hline DR2 & $.458 * *$ & $.096^{*}$ & $.036^{*}$ \\
\hline
\end{tabular}

$F(7,51)=10.515 p<.0005 R 2=.591$

* Beta is significant at the 0.05 level

**Beta is significant at the 0.01 level

Although in both models the relationship between the variables and the turnover intention were significant, in the findings for married women the three-way interaction was negative, whereas in the model for unmarried the three-way interaction was positive. Meaning that for married nurses, the three-way interaction significantly decreased their turnover intention. However, for unmarried women the three-way interaction did not significantly decreased turnover intention.

The three-way interaction was plotted to display the relation among the variables, examining the relationship between $X$ and $\mathrm{Y}$, at high and low values of $\mathrm{Z}$ and $\mathrm{W}$, as discussed by Aiken \& West, 1991. Building on the work of Aiken and West (1991), the straight forward way to visualize and interpret the three-way interaction is to compare and contrast the slopes of the four lines. First, the regression equation which includes a three-way interaction variable was created. In this formula, $\mathrm{Y}$ is the independent variable (Turnover Intention), $X$ the predictor variable (WFC), with Z (STRESS) and W (FSSB) being the two moderator variables.

The influence of FSSB on the relationship among WFC, stress, and turnover intention among married nurses is illustrated by the two graphs in Figure 1. For married nurses, turnover intention decreased as the conflict increased with high support especially with higher levels of stress. With low FSSB, the turnover intention increases as the conflict increased especially with higher levels of stress (Figure 1).

Figure: 1 Slopes for The three-way interaction effects of FSS and Stress on the relationship between WFC and T (Married nurses).

As displayed in Figure 2, there is a no influence of FSSB on the relationship between WFC and turnover intention among unmarried nurses. For unmarried nurses with high stress as well as with low stress, turnover intention increased as WFC increased with both high FSSB and low FSSB. 

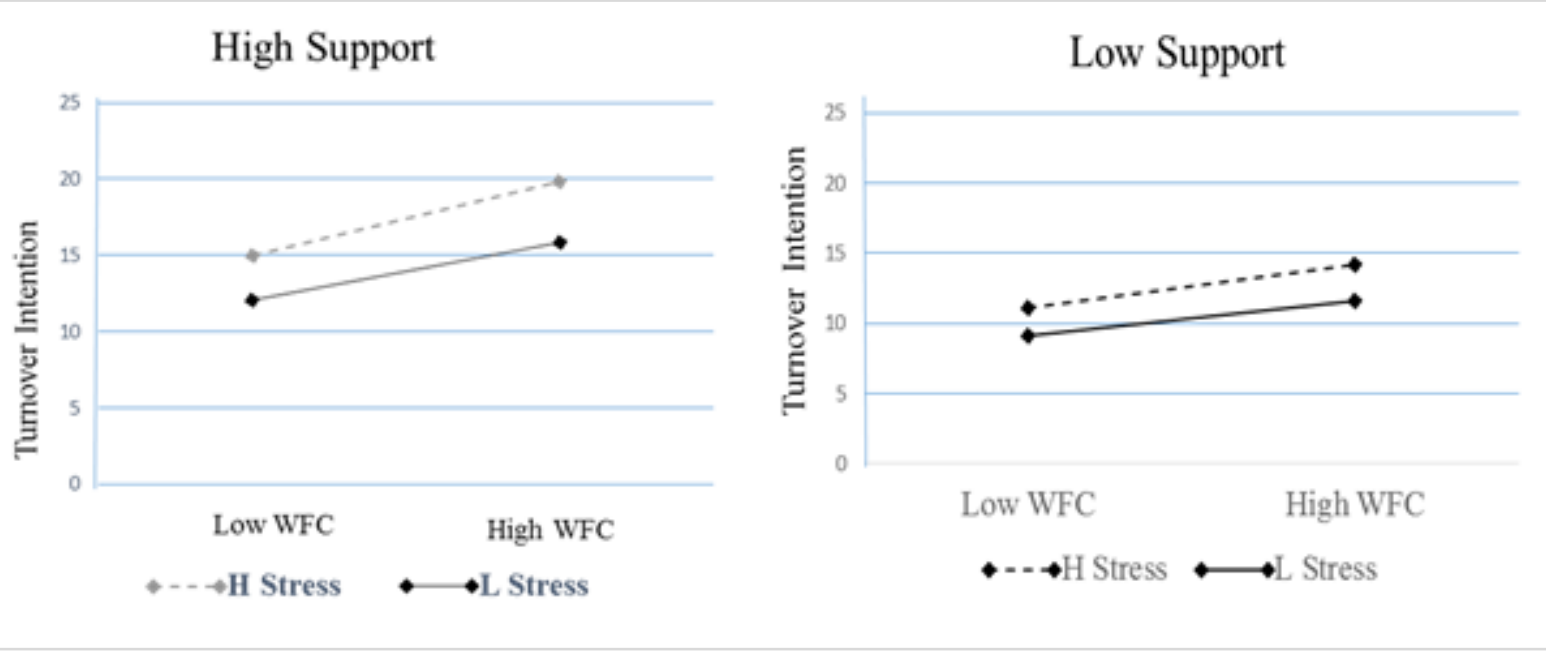

Figure 2: Slopes for The three-way interaction effects of FSS and Stress on the relationship between WFC and TI (Not Married nurses).

The purpose of this study was to evaluate the moderating effect of FSSB on the relationship among WFC, stress and turnover intention. While considering the influence of culture in Saudi Arabia, it was proposed that the moderation effect of FSSB would be different based on the marital status of nurses. The reported main effects of WFC, Stress, and FSSB on the level of turnover intention were consistent with earlier research; WFC and Stress have positive correlation with turnover intention, while FSSB has negative correlation with turnover intention (Shockley \&Allen, 2013; Li, Shaffer, \& Bagger, 2015 Kalidass \& Bahron, 2015; Nohe \&Sonntag, 2014).

With further examination using the interactions among the independent variables, the study hypothesis was supported; the FSSB buffered the influence of WFC and stress on turnover intention among married nurses. Despite the higher levels of WFC and stress, married nurses who reported higher FSSB had lower turnover intention. Whereas married nurses who reported high level of WFC and Stress but less FSSB were more intent to leave. These findings may be interpreted that for married women, it is essential to have FSSB in the workplace to reduce the negative influence of WFC and stress on their intention to leave. The plotted simple slopes (Fig. 1) confirmed this influence; with high FSSB the relationship between WFC and turnover intention was weakening even with high stress. The result of this study was consistent with previous research that found similar moderation effect of FSSB (Nohe \& Sonntag, 2014; Almeida et al., 2016).

Considering the defined role of married women in Saudi Arabia, this study revealed that married nurses were more intent to leave that non-married nurses. Therefore, the result of this study indicated that FSSB can help reduce married nurses' intention to leave by buffering the influence of WFC and Stress. This study was the first study conducted in Saudi Arabia, so there were no other studies to compare the results. However, a growing body of literature has highlighted the importance of FSSB as a key resource for employees to balance work and family demands (Almeida et al., 2016; Crain et al., 2014; Nohe \&Sonntag, 2014; Hammer et al., 2011).

\section{Nursing implications}

With the current shortage of nursing workforce, it is important to maintain nurses in the workplace and investigate ways to reduce turnover. Several studies describe associations between registered nurses' perception of their practice environment and nurse outcomes such as job satisfaction, RN quality of care (Moore,
2014), and turnover intention; as well as patient outcomes such as mortality and failure to rescue (Van Bogaert et al., 2010). Nursing leaders and policy makers need to investigate ways to create a more supportive work environment for nurses to enhance organizational and patients' outcomes.

This study finding suggested important practical implications for nursing; an obvious implication is that nurses' turnover intention could be reduced by reducing their WFC. Therefore, healthcare organizations may benefit from offering formal work-family policies, such as flexible work schedules, that could help nurses in balancing work and family demands (Ryan \& Kossek, 2008; Sutton \& Noe, 2005). Further, it may be beneficial to offer educational programs and workshops for nurses to improve specific skills to balance the demands of work and family (Baltes \& Heydens-Gahir, 2003).

Another implication is that the relationship between WFC and turnover intention could be reduced by FSSB. In this regard, healthcare organizations are advised to develop and adopt strategies to promote FSSB. For example, official guidelines for supervisors to use supportive behaviors related to employees' family issues. Furthermore, implementing training programs for supervisors to enhance their ability to address work-family issues and to be more sensitive to WFC have been shown to be beneficial for employee health outcomes (Hammer et al., 2011). Further interventional and longitudinal studies are recommended to evaluate the effectiveness of such supervisor training programs.

The study findings suggest the importance of cultural context in studying WFC. Therefore, further studies are recommended to explore the relationships between WFC and other variables that could be different from one culture to another. For example, family perception about working women, working night shifts, and the nature of workplace need further examination. In a conservative gender-segregated society such as Saudi Arabia these variables could change the level of WFC and its consequences.

Although positive effects of support are promising in reducing nursing turnover intention, there are limited studies examining the impact of FSSB among nurses' population. More research in this area is strongly recommended to identify implications for nursing especially with the shortage of registered nurses in the workforce. Understanding what nurses perceive as specific supportive behaviors as demonstrated by their supervisors can inform future plans designed to promote supervisors' behaviors that lead to nursing retention.

\section{Conclusion}


Managing work and family demands is a huge challenge for many employees. With the defined domestic role and higher expectations of married women in the Saudi society, married nurses are more exposed to WFC and stress. The results of this study suggested the importance of providing FSSB to help married nurses reduce the consequences of WFC and stress. The FSSB could be the tool to facilitate married nurses perform their professional duties without compromising their family responsibilities. Nurses' turnover is a complex issue that may require multiple prevention strategies; however, enhancing FSSB could be a key resource for maintaining a positive workplace environment and reducing the turnover intention.

\section{References}

[1] Alyaemni, A., et al. (2013). Gender Inequities in Health: An Exploratory Qualitative Study of Saudi Women's Perceptions. Women Health, 53(7), 741.

[2] Al-Tarrah, Ali (2010). Women in the Development of Kuwait. Digest of Middle East Studies 2,1-33.

[3] Almeida, D., et al. (2016). Supervisor Support Buffers Daily Psychological and Physiological Reactivity to Work-to-Family Conflict. Journal of Marriage and Family, 78(1), 165179.

[4] Amstad FT, Meier LL, Fasel U, Elfering A, Semmer NK (2011) A metaanalysis of work family conflict and various outcomes with a special emphasis oncrossdomain versus matching domain relations. Journal of Occupational Health Psychology 16: 151-169.

[5] Aryee, S., Fields, D. and Luk, V. (1999), "A crosscultural test of a model of the work-family interface", Journal of Management, Vol. 25 No. 4, pp. 491-512

[6] Bahudhailah, M. (2015). Exploring the Impact of Flexible Work on Work life Balance for Women in Saudi Arabia. IDEAS Working Paper Series from RePEc, IDEAS Working Paper Series from RePEc, 2015.

[7] Buxton, O. M., Klein, L. C., Whinnery, J., Williams, S., \& McDade, T. (2013). Biomarkers in work and family research. In J. G. Grzywacz \& E. Demerouti (Eds.), new frontiers in work and family research (pp. 170-190).East Sussex, UK: Psychology Press.

[8] Bogaert, S. Clarke, E. Roelant, H. Meulemans, P. Van de Heyning. (2010), Impacts of unit level nurse practice environment and burnout on nurse-reported outcomes: a multilevel modelling approach. Journal of Clinical Nursing, 19 (11-12) pp. 16641674

[9] Cohen, S., Gottlieb, B. H., \& Underwood, L. G. (2000). Social relationships and health. In $\mathrm{S}$.

[10] Crain, TL, Hammer LB, Bodner T, Kossek EE, Moen P, et al. (2014) Work-family conflict, family-supportive supervisor behaviors (FSSB), and sleep outcomes. Journal of Occupational Health Psychology 19: 155-167.

[11] Coyle, E., Van Leer, F., Schroeder, E., \& Fulcher, K. (2015). Planning to Have It All: Emerging Adults' Expectations of Future Work-Family Conflict. Sex Roles, 72(11), 547-557.

[12] Charles, S. T., Piazza, J. R., Mogle, J., Sliwinski, M. J., \& Almeida, D. M. (2013). The wear and tear of daily stressors on mental health. Psychological Science, 24, 19. Doi:10.1177/0956797612462222

[13] Eby, W.J. Casper, A. Lockwood, C. Bordeaux, A. Brinley (2005). A retrospective on work and family research in IO/OB: A content analysis and review of the literature. Journal of Vocational Behavior, 66, pp. 124 197

[14] Elamin, Abdallah M. \& Katlin Omair. (2010). Males' attitudes towards working females in Saudi Arabia. Personnel Review, 39(6), 746-766.

[15] Fargues, P. (2005), 'Women in Arab Countries: Challenging the Patriarchal System?' Reproductive Health Matters, 13, 25, 43-48.

[16] Greenhaus, J. H., Allen, T. D., \& Spector, P. E. (2006). Health consequences of work-family conflict: The dark side of the work-family interface. In P. L. Perrewe \& D. C.

[17] Grzywacz, J. G. (2000). Work-family spillover and health during midlife: Is managing conflict everything? American Journal of Health Promotion, 14, 236-243.

[18] Hoobler, JM, Wilson M (2010) Do workers who experience conflict between the work and family domains hit a "glass ceiling"?: A metaanalytic examination. Journal of Vocational Behavior 77: 481494.

[19] Kossek, S. Pichler, T. Bodner, L.B. Hammer. (2011).Workplace social support and work family conflict: A meta-analysis clarifying the influence general and work-family specific supervisor and organizational support. Journal of Personnel Psychology,

[20] Krouse, S. S. and Afifi T. D. (2007). Family-to-work Spillover Stress: Coping Communicatively in the Workplace. Journal of Family Communication 7(2), 85122.

[21] Kelly, E.L. Moen, Oakes, J.M. Fan, W. Okechukwu, C. Davis, K.D. Mierzwa. F. (2014). Changing work and work-family conflict evidence from the work, family, and health network. American Sociological Review.

[22] Kalidass A, Bahron A. (2015). The Relationship between Perceived Supervisor Support, Perceived Organizational Support, Organizational Commitment and Employee Turnover Intention. International Journal of Business Administration, 6, (5).

[23] Lal, D. (2012, October 15). Saudi Arabia: Working Women in Saudi Arabia. Yes, You Read It Right. Women's Feature Service, p. N_A.

[24] Leineweber, et al. (2014). Hospital organizational factors influence work-family conflict in registered nurses: Multilevel modeling of a nation-wide cross-sectional survey in Sweden. International Journal of Nursing Studies, 51(5), 744-751.

[25] Mroczek, D.,et al. (2015).Emotional reactivity and mortality: Longitudinal findings from the VA Normative Aging Study. Journals of Gerontology, Series B: Psychological Sciences and Social Sciences, 70(3), 398406.

[26] Nazzah, N. (2004), "Working national women will be released from duty by 4pm", Gulf News, March 1, p.3.

[27] Nohe, C., \& Sonntag, K. (2014). Work-family conflict, social support, and turnover intentions: A longitudinal study. Journal of Vocational Behavior, 85(1), 1.

[28] Piazza, J. R., et al. (2013). Affective reactivity to daily stressors and long-term risk of reporting a chronic physical health condition. Annals of Behavioral Medicine, 45, 110-120.

[29] Parker, K., \& Wang, W. (2013). Modern parenthood: Roles of moms and dads converge as they balance work 
and family. Pew Research Center: Social and Demographic Trends.

[30] Sha'aban, B. (1996), "The status of women in Syria", in Sabbagh, S. (Ed.), Arab Women: Between Defiance and Restraint, Olive Branch Press, New York, NY, pp. 54-61.

[31] Shockley, T.D. Allen Episodic. (2013). work-family conflict, cardiovascular indicators, and social support: An experience sampling approach Journal of Occupational Health Psychology, 18 (3), p. 262

[32] Shockley, K. M., \& Allen, T. D. (2013). Episodic workfamily conflict, cardiovascular indicators, and social support: An experience sampling approach. Journal of Occupational Health Psychology, 18, 262-275.

[33] Shallal, M. (2011). Job Satisfaction among Women in the United Arab Emirates. Journal of International Women's Studies, 12(3), 114-134.

[34] Vanagas, G., S. Bihari-Axelsson, and V. Vanagiene (2004). Do Age, Gender and Marital Status Influence Job Strain Development for General Practitioner/0410/0410$14 \mathrm{e}$.

[35] Buffering effects of supervisor support on retaining female nurses on Saudi Arabia. 